\title{
Design of an active anti-roll bar for off-road vehicles
}

\author{
S. Gosselin-Brisson ${ }^{\mathrm{a}}$, M. Bouazara ${ }^{\mathrm{a}, *}$ and M.J. Richard ${ }^{\mathrm{b}}$ \\ ${ }^{a}$ Applied sciences, University of Quebec at Chicoutimi, Saguenay, QC, Canada, G7H-2BI \\ ${ }^{\mathrm{b}}$ Mechanical Engineering, Laval University, QC, Canada, G1K-7P4
}

Received 9 August 2007

\begin{abstract}
This paper presents a comparison of performance between a passive and an active anti-roll bar. Off-road vehicles are subject to large input road motion and appreciable lateral forces, making anti-roll bars desirable. A four DOF linear model is used to represent an independent suspension and to design the controller. For every case the performance is evaluated for severe road input perturbation and lateral acceleration. A method is presented to illustrate the compromise between stability and comfort inherent in passive anti-roll bar selection. This method was used to select a realistic anti-roll bar stiffness. The active anti-roll bar was designed using full state feedback optimal controller. A simplification of the active system is proposed to reduce the number of measurements and eliminate the need for an optimal observer. The results show a superior performance in ride and handling for the active controller in the frequency range of interest. The addition of filters is proposed to maximize controller efficiency and to reduce associated problems.
\end{abstract}

\section{Introduction}

Vehicle suspension plays a key role in passenger comfort and vehicle stability. To improve these performances a wide variety of solutions are used and are subject to research, the main ones being passive, semi active and fully active suspensions. Since the beginning of the century, passive suspensions are used and continuous improvements have been made to them through continuous research. Advances are so important that today most of the experts think the passive systems can hardly be improved [1-4]. However, Chalassani proved that a fully active suspension could deliver a better ride and handling performance than a passive system [12]. For the last decades an increasing number of researchers have worked on the design and testing of different active suspension systems with positive results. At the moment these studies are conducted on road vehicles and use simplified suspension models. Most of the experiments [1-4] use a quarter car vehicle model, which ignores the sprung mass roll but has a great effect on the comfort and stability of the vehicle.

An off-road vehicle, more than an automobile, is subject to severe road perturbations. A normal off-road vehicle suspension is relatively soft with large suspension displacements to provide an acceptable comfort zone for passengers. To provide enough clearance, the centre of gravity (CG) is relatively high. The combination of soft suspension and high CG decreases the stability during cornering, increases the sprung mass roll angle and ultimately the chances of a roll over. On the other hand, stiffer suspension greatly reduces passenger comfort on rough roads. To resolve this dilemma, active anti-roll bar (ARB) systems have been developed for automobiles [1] but their application in off-road vehicles is limited. For the above mentioned reasons, the active ARB has a huge potential to improve both comfort and stability, making off-road vehicles safer.

This paper presents the design of an active ARB controller to improve both vehicle stability and passenger comfort. To objectively evaluate the active system, it is compared to a passive suspension with and without a passive anti-roll

\footnotetext{
*Corresponding author. E-mail: mbouazar@uqac.ca.
} 
Table 1

Suspension parameters

\begin{tabular}{llll}
\hline Symbol & Parameter & Value & Units \\
\hline $\mathrm{m}_{s}$ & Sprung mass & 600 & $\mathrm{~kg}$ \\
$\mathrm{I}_{s}$ & Sprung mass inertia & 1300 & $\mathrm{~kg} \mathrm{~m}$ \\
$\mathrm{~m}_{u R}=\mathrm{m}_{u L}=\mathrm{m}_{u}$ & Unsprung mass & 50 & $\mathrm{~kg}$ \\
$\mathrm{k}_{s R}=\mathrm{k}_{s L}=\mathrm{k}_{s}$ & Suspension stiffness & 20000 & $\mathrm{~N} / \mathrm{m}$ \\
$\mathrm{c}_{s R}=\mathrm{c}_{s L}=\mathrm{c}_{s}$ & Suspension damping & 1500 & $\mathrm{Ns} / \mathrm{m}$ \\
$\mathrm{k}_{t R}=\mathrm{k}_{t L}=\mathrm{k}_{t}$ & Tire stiffness & 100000 & $\mathrm{~N} / \mathrm{m}$ \\
$\mathrm{L}$ & Track width & 2 & $\mathrm{~m}$ \\
$\mathrm{~h}$ & Distance between CG and RC & 1 & $\mathrm{~m}$ \\
\hline
\end{tabular}

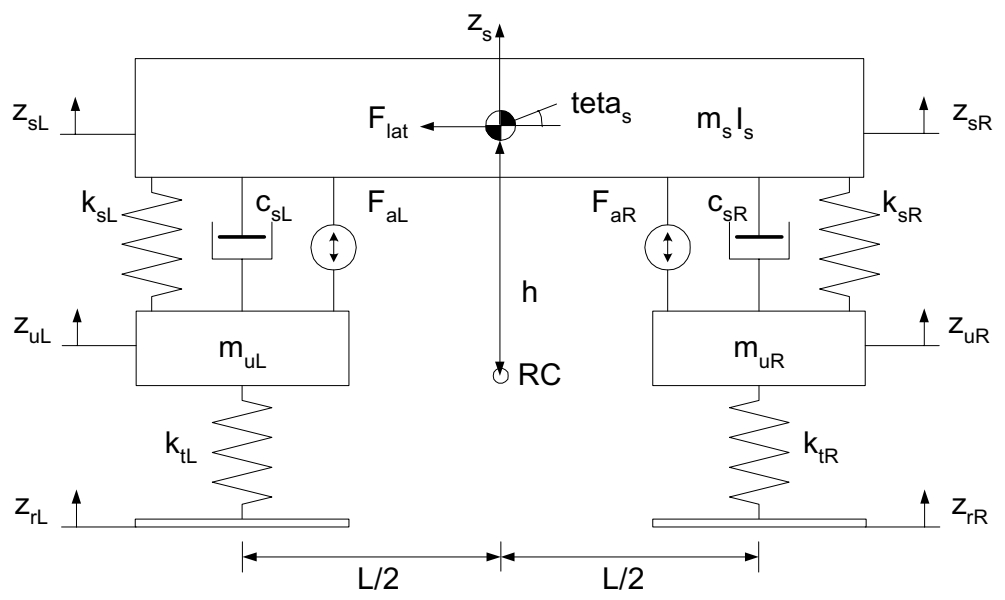

Fig. 1. Vehicle suspension model.

bar. The passive anti-roll bar stiffness was chosen by selecting appropriate parameters: suspension deflection, roll angle and angular acceleration. The active system is designed using the same criteria and the classic Riccatti equation. A simplification of the controller is proposed to eliminate the need of an optimal observer. The performances of all the proposed solutions are compared and analyzed. Final modifications are proposed to maximize the efficiency of the active system.

\section{Vehicle model}

The model represents the front view of a vehicle suspension system as shown in Fig. 1. It includes four degrees of freedom (DOF) to model an independent suspension instead of the three DOF commonly used in the literature [5-7] where the right and left unsprung masses are modeled by one axle only.

For this work we assume that the vehicle has the same parameters on the right and left sides, which is generally true. Model parameters in Table 1 represent a medium size off-road vehicle.

Tire damping is ignored, as is usually the case in the literature [1-4]. To keep a symmetrical vehicle the sprung mass gravity centre (CG) is in the middle of the track. The track has a length $\mathrm{L}$ so the distance between each tire and the sprung mass gravity centre is $\mathrm{L} / 2$.

During roll motion the vehicle rotates around the sprung mass roll centre (RC) [1]. The position of this point is a function of suspension geometry, vehicle ride height and roll angle [8]. As the sprung mass moves in roll and heave the RC movement amplitude depends on the suspension geometry. As a general rule large RC movement could compromise stability and should be avoided. In this study we keep a fixed RC position to simplify the model [8]. Parameter h corresponds to the distance between the sprung mass CG and the sprung mass RC. $\mathrm{F}_{a R}$ and $\mathrm{F}_{a L}$ are the forces applied by the left and right actuators to control the vehicle sprung mass roll. 


\section{Suspension model}

As stated before, three different cases are analyzed: passive suspension without anti-roll bar, passive suspension with passive anti-roll bar and passive suspension with active anti-roll bar. Each case is modeled by different roll control force equations.

The passive suspension without anti-roll bar model is an extension of the widely used two DOF quarter car model. The suspension is composed of a spring and a damper for each side of the vehicle. In this case the roll control forces are eliminated, because the only roll stiffness is provided by the suspension spring and damper. Here:

$$
\begin{aligned}
& F_{a R}=0 \\
& F_{a L}=0
\end{aligned}
$$

The passive suspension with passive anti-roll bar model is representative of most common vehicles in use today. To reduce roll motion, an anti-roll bar is added to the suspension system, increasing the total roll stiffness. The ARB is usually made of steel and acts like a spring connected to the right and left unsprung masses. The ARB force is a function of the difference between right and left suspension deflection. The force is applied by the bar on each side of the vehicle so that the left force has the same magnitude and the opposite direction as the right one. For an ARB stiffness $\mathrm{k}_{A R B}$, the force corresponds to:

$$
F_{A R B}=-k_{A R B}\left[\left(z_{s R}-z_{u R}\right)-\left(z_{s L}-z_{u L}\right)\right]
$$

To model the passive anti-roll bar the forces $\mathrm{F}_{a R}$ and $\mathrm{F}_{a L}$ are given by Eq. (3).

$$
\begin{aligned}
& F_{a R}=F_{A R B}=-k_{A R B}\left[\left(z_{s R}-z_{u R}\right)-\left(z_{s L}-z_{u L}\right)\right] \\
& F_{a L}=-F_{A R B}=k_{A R B}\left[\left(z_{s R}-z_{u R}\right)-\left(z_{s L}-z_{u L}\right)\right]
\end{aligned}
$$

For a passive suspension with an active anti-roll bar, the first step in the design is to locate the actuator. Generally a pneumatic or hydraulic piston [1] is used; therefore it is modeled by a controllable force. This actuator is placed in series with a stiff anti-roll bar. The force is applied with the same magnitude but in opposite directions as for the passive ARB. However, the force magnitude $\mathrm{F}_{a}$ is calculated by the controller. As a result the forces are applied as in equation 4.

$$
\begin{aligned}
& F_{a R}=F_{a} \\
& F_{a L}=-F_{a}
\end{aligned}
$$

Three different inputs can be applied to the models: lateral force, left and right road motion. The application of left and right road motion with equal phase and amplitude results in pure vertical motion similar to the quarter model behaviour. Since this model is extensively studied in the literature, it isn't detailed in this paper. On the other hand, the excitation of a single wheel gives valuable indication on how the system transfers the road perturbation from one side to the other. Since the ARB has a noticeable impact on this transfer, the first case studied is the application of a road motion input to a single wheel. Since the side of the excitation application doesn't change the response amplitude, the input is applied at the right tire/road interface.

In off-road real life use, cornering manoeuvres are unavoidable. The change in trajectory is accomplished by creating a lateral acceleration. Since the response of the vehicle's suspension during these manoeuvres is critical for stability, lateral acceleration input is the second case studied in this work.

The roll motion is caused by the vehicle's lateral acceleration, which depends on the speed and corner radius. In a manner similar to the road perturbation, the direction of the force only changes the direction of the response since the vehicle is symmetrical. For this work a right turn is simulated resulting in a left acceleration creating a force at the sprung mass CG. Since the force is applied at a distance $\mathrm{h}$ from the rotation center, a moment $\mathrm{M}_{s}$ is created on the sprung mass with a magnitude given by equation 5 .

$$
M_{s}=a_{\text {lat }} m_{s} h
$$

The system is modeled using the state space variable formulation. System variables and equations are given by equations 6 to 11 . 


$$
\begin{aligned}
& x_{1}=z_{s R}-z_{u R} \\
& x_{2}=z_{u R}-z_{r R} \\
& x_{3}=\dot{z}_{u R} \\
& x_{4}=z_{s L}-z_{u L} \\
& x_{5}=z_{u L}-z_{r L} \\
& x_{6}=\dot{z}_{u L} \\
& x_{7}=\dot{z}_{s} \\
& x_{8}=\dot{\theta}_{s} \\
& x_{9}=\theta_{s} \\
& \dot{x}=A x+B u+L_{r R} z_{r R}+L_{a} a_{l a t}
\end{aligned}
$$

$A=\left[\begin{array}{ccccccccc}0 & 0 & -1 & 0 & 0 & 0 & 1 & \frac{L}{2} & 0 \\ 0 & 0 & 1 & 0 & 0 & 0 & 0 & 0 & 0 \\ \frac{k_{s}}{m_{u}} & \frac{-k_{t}}{m_{u}} & \frac{-c_{s}}{m_{u}} & 0 & 0 & 0 & \frac{c_{s}}{m_{u}} & \frac{L c_{s}}{2 m_{u}} & 0 \\ 0 & 0 & 0 & 0 & 0 & -1 & 1 & \frac{-L}{2} & 0 \\ 0 & 0 & 0 & 0 & 0 & 1 & 0 & 0 & 0 \\ 0 & 0 & 0 & \frac{k_{s}}{m_{u}} & \frac{-k_{t}}{m_{u}} & \frac{-c_{s}}{m_{u}} & \frac{c_{s}}{m_{u}} & \frac{-L c_{s}}{2 m_{u}} & 0 \\ \frac{-k_{s}}{m_{s}} & 0 & \frac{c_{s}}{m_{s}} & \frac{-k_{s}}{m_{s}} & 0 & \frac{c_{s}}{m_{s}} & \frac{-2 c_{s}}{m_{s}} & 0 & 0 \\ \frac{-L k_{s}}{2 I_{s}} & 0 & \frac{L c_{s}}{2 I_{s}} & \frac{L k_{s}}{2 I_{s}} & 0 & \frac{-L c_{s}}{2 I_{s}} & 0 & \frac{-L^{2} c_{s}}{2 I_{s}} & 0 \\ 0 & 0 & 0 & 0 & 0 & 0 & 0 & 1 & 0\end{array}\right]$

$B=\left[\begin{array}{c}0 \\ 0 \\ \frac{-1}{m_{u}} \\ 0 \\ 0 \\ \frac{1}{m_{u}} \\ 0 \\ L \\ \frac{L}{I_{s}} \\ 0\end{array}\right]$

$$
L_{r R}=\left[\begin{array}{c}
0 \\
-1 \\
0 \\
0 \\
0 \\
0 \\
0 \\
0 \\
0
\end{array}\right]
$$

$$
L_{a}=\left[\begin{array}{c}
0 \\
0 \\
0 \\
0 \\
0 \\
0 \\
0 \\
\frac{m_{s} h}{I_{s}} \\
0
\end{array}\right]
$$


In order to compare different suspensions, the same performance criteria are used. The suspension system has to assure vehicle stability and passenger comfort [10]. In the case of off-road vehicles three measurements are considered representative: suspension deflection, roll angle and angular acceleration. The importance of each criterion is detailed in the following section.

Primarily, the designed suspension system has to be applicable on a real vehicle. Off-road vehicles have a large suspension travel compared to the average automobile. This is an essential feature to cope with the high amplitude bumps on rough roads. Even with this large suspension travel, it is imperative to reduce the suspension deflection for two reasons. It will ensure that the available travel is efficiently used and that chances of suspension bottoming are reduced.

For an automotive vehicle, the risk of roll over is small and stability is improved by reducing tire deflection. Reduced deflection gives a more stable tire load resulting in a more stable and predictable handling [6,7]. For SUVs (Sport Utility Vehicle) and off-road vehicles the centre of gravity is higher and the suspension is softer, making the risk of roll over greater. For off-road vehicles subjected to severe road perturbation, we consider the limiting factor for stability is the risk of roll over instead of the tire deflection as reported in other papers [6,7]. Vehicle stability is measured using the sprung mass roll angle. Thus the second objective of this research is minimizing the sprung mass roll angle to improve stability.

Comfort is a difficult parameter to evaluate since it is a personal human experience and each individual has different opinions about the same ride [11]. It is generally accepted that the minimisation of the sprung mass vertical acceleration improves passenger comfort in a two DOF quarter car model [10]. Norm ISO-2631 uses vertical acceleration to define the human limits for vibrations. For a model including roll motion it is also critical to minimise the sprung mass angular acceleration. In this work the vertical acceleration is similar for each system so the analysis is focused on the angular acceleration of the sprung mass to quantify passenger comfort.

For an active suspension the actuator is another limiting factor. The force is provided by a compressor or alternator thus requiring power. To keep power consumption at a realistic value, the actuator's force should be minimised [10]. The dynamic response will show a delay as frequency increases for a hydraulic or pneumatic actuator. This will compromise the high frequency operation of the active system as detailed later.

The design of the passive ARB consists of choosing an optimal stiffness. As will be shown later, there is no optimal stiffness, because it is impossible to improve all criteria at the same time. The proposed solution is to determine the relative gain/loss of performance as the ARB stiffness increases. Once this information is available, the designer can make a better decision.

The MS (Mean Square) value of the amplification is developed in Eq. (12). This value is exploited to represent the performance of one criterion for all the frequencies of interest [12]. Since the goal is to minimise a given performance criteria " $\mathrm{y}$ ", the smaller the MS value of the transfer function $\mathrm{H}_{y}$, the better the performance. Transfer function $\mathrm{H}_{y}$ is the frequency response of a measurement " $\mathrm{y}$ " to a system input.

$$
E\left[y^{2}\right]=S_{0} \int\left|H_{y}(\omega)\right|^{2} d \omega
$$

In this work the two inputs studied are road motion and lateral acceleration. To keep the measurements in the useful frequency range, the integration is limited to frequencies between 0.1 and $30 \mathrm{~Hz}$ [9] for road motion input. This range includes low frequencies from close to steady state to up to $30 \mathrm{~Hz}$, which is beyond unsprung mass resonance. Frequencies between 0.1 and $5 \mathrm{~Hz}$ are used for lateral acceleration input. Road input includes low frequencies from close to steady state to up to the maximum frequency measured by data acquisition [13].

The choice of ARB stiffness $\mathrm{k}_{A R B}$ implies the minimisation of all the performance criteria. To represent the global performance, roll angle, angular acceleration and suspension deflection are compared. For a road motion input the right and left suspension deflection aren't equal so the maximum is used to represent the vehicle's suspension deflection [14]. For cornering, the right and left suspension deflections are equal with opposite directions, so the magnitude is the same.

Good suspension design is a compromise between conflicting criteria appropriate to the vehicle type. Because the suspension reacts to road imperfections and lateral acceleration, one graph is plotted for each input.

For a right wheel road input the suspension deflection increases with anti-roll bar stiffness up to $17000 \mathrm{~N} / \mathrm{m}$, then slowly decreases. As the ARB stiffness increases, the suspension stiffness also increases, thus increasing the critical 

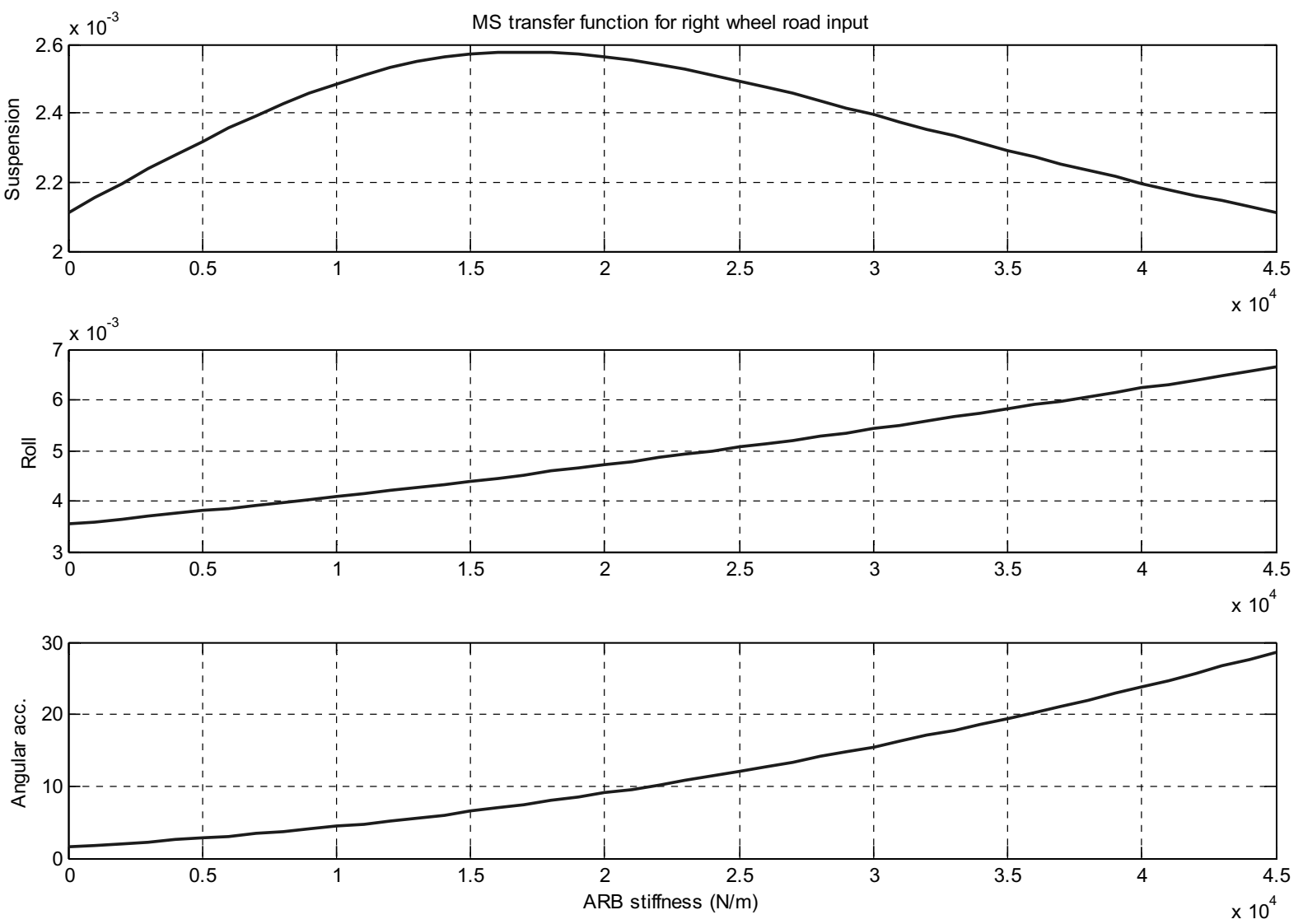

Fig. 2. RMS values of transfer function for right road input.

damping value. Since the suspension damping keeps a fixed value, the suspension damping coefficient is reduced, resulting in a sharper but higher resonance peak. This higher amplification with resonance causes the higher MS value seen in the graph. The phenomenon can be seen in Fig. 3 showing the frequency response of suspension deflection for different ARB stiffness.

Increased suspension stiffness has a similar effect on both the roll and angular acceleration frequency responses. In these cases the resonance frequency increases for a fixed damping, increasing the resonance amplitude. For these two criteria the progression is more linear as can be seen in Fig. 2. For an independent suspension the increase in ARB stiffness causes increased suspension stiffness. For the same road motion applied at the right wheel, a stiffer suspension will cause less suspension deflection but will increase the motion of the sprung mass on the right side, leading to an increase in roll angle and angular acceleration. These results confirm that for maximum ride and handling performance the ARB stiffness should be reduced when there is a roadinput.

Figure 4 shows the three performance criteria when a lateral acceleration excites the system. The suspension deflection decreases smoothly as ARB stiffness increases. The purpose of the ARB is to increase vehicle roll stiffness. Thus the reduction in suspension deflection is an expected consequence.

For the same reason the roll is also reduced as ARB is stiffened to $15000 \mathrm{~N} / \mathrm{m}$. Figure 5 details the frequency response of the roll for a lateral acceleration input. In the low frequency range close to steady state the reduction in roll decreases but the resonance increases as ARB stiffness increases. The soft tires used on off-road vehicles limit the efficiency of a stiff ARB in reducing roll since they are installed in series with the suspension springs. For ARB stiffer than $15000 \mathrm{~N} / \mathrm{m}$ the benefits of reduced low frequency amplification are smaller than the increase of resonance which is controlled by the square function in Eq. (12). This explains the reduction in roll from 0 to 15000 N/m visible on Fig. 4 and the increase of roll for ARB stiffness above 15000 N/m. 


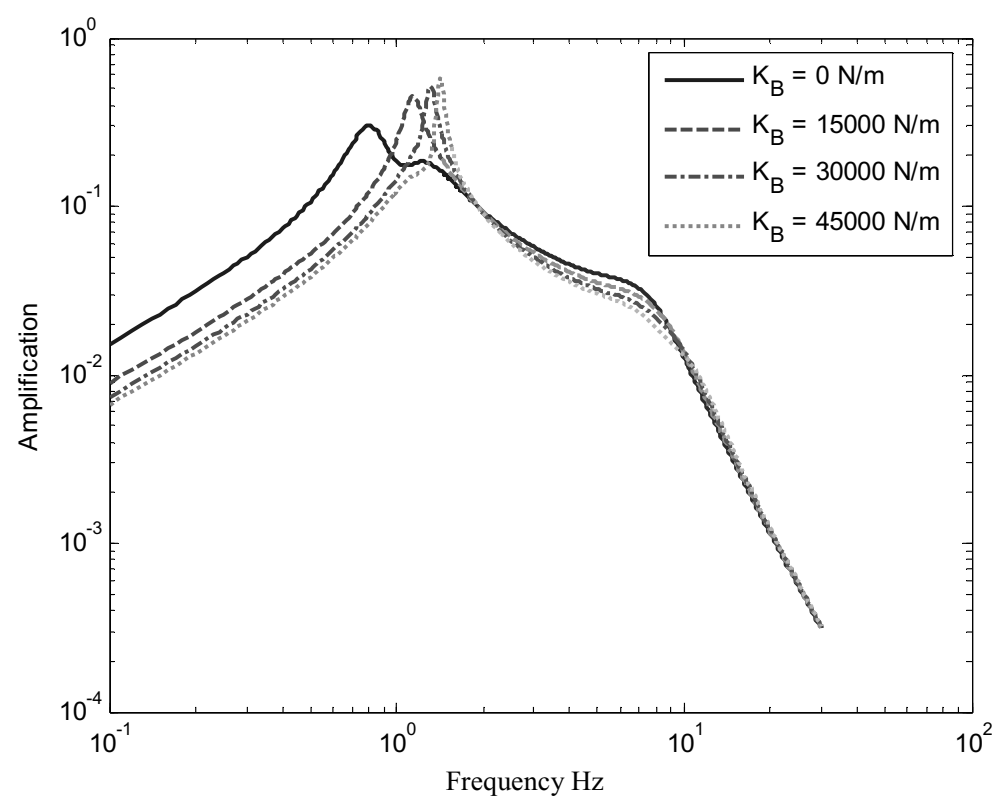

Fig. 3. Suspension deflection of passive suspension with passive ARB.

Table 2

Weight for performance index

\begin{tabular}{lll}
\hline Weight & Performance criteria & Value \\
\hline$\rho_{s}$ & Suspension deflection & 10 \\
$\rho_{\text {tetas }}$ & Sprung mass roll angle & 10000 \\
$\rho_{\text {ddtetas }}$ & Angular acceleration & 1 \\
$\rho_{F}$ & Actuator force & 0.000001 \\
\hline
\end{tabular}

The increase in ARB stiffness has a reverse effect on angular acceleration, which increases steadily with ARB. For the low frequency range the system is close to steady state and the acceleration is similar for all ARB stiffness. However, as roll stiffness increases with a fixed damping value, the consequence is an increased resonance frequency amplitude. These results show that ARB increases stability during cornering by reducing roll angle but decreasing passenger comfort.

With this in mind the designer has to make a compromise in the choice of ARB stiffness. All the measured criteria have different amplitudes and importance. The initial values and the slope of the different curves indicate the relative change caused by variation of ARB stiffness. The advantage of the presented method is that the gain/loss in performance can be clearly seen in Figures 2 and 4. The graphs detail the behaviour of the different criteria for different inputs allowing the designer to place more emphasis on the most important criteria according to the vehicle's requirements.

The main goal for the present off-road vehicle study is to reduce roll angle with an acceptable reduction in vehicle comfort. An ARB stiffness of $7500 \mathrm{~N} / \mathrm{m}$ is considered reasonable since it noticeably reduces roll during cornering without loosing too much comfort.

\section{Design of the active roll bar controller}

As previously shown, it isn't possible to improve both ride and handling for all the inputs using a passive anti-roll bar. The use of an active system is a potential solution for this dilemma. The hardware was briefly described in Section 3. Interested readers can consult [13] for details on actuator dynamics and linearization.

The design of the active ARB is centred on actuator control law definition. This is done with the same performance criteria as those used for the passive ARB. To keep the force used in a realistic range the actuator force is added to 

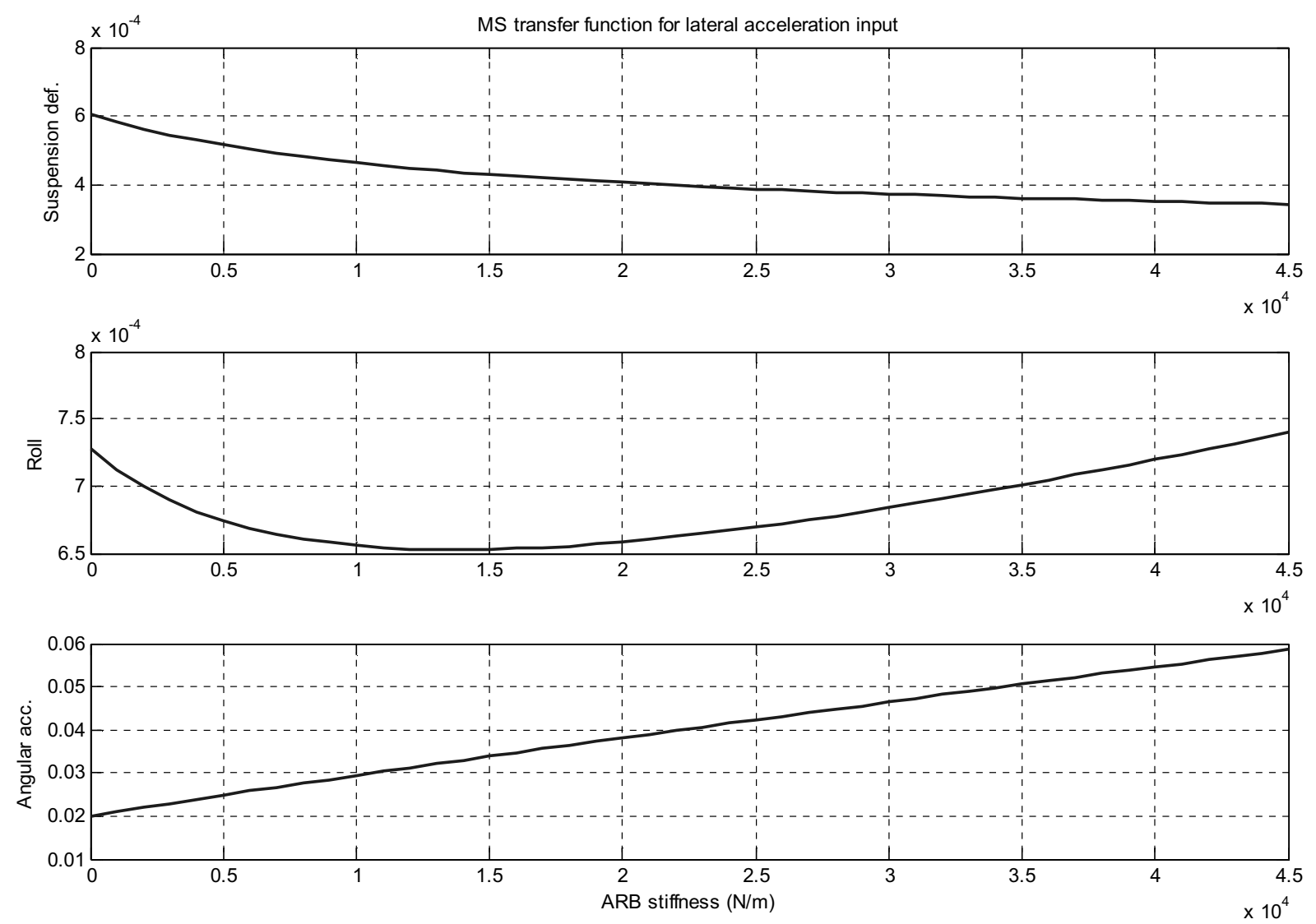

Fig. 4. RMS values of transfer function for acceleration input.

the performance criteria. The controller is synthesised using the algebraic Ricatti equation (ARE) to obtain a "Full State Feedback" (FSF) control law. Before solving the ARE, a performance index $\mathrm{J}$ is defined as a function of the system state variables and control input. Equation 13 is used in this study.

$$
J=\frac{1}{T} E\left[\int\left(\rho_{s}\left(z_{s R}-z_{u R}\right)^{2}+\rho_{s}\left(z_{s L}-z_{u L}\right)^{2}+\rho_{\text {tetas }} \theta_{s}^{2}+\rho_{\text {ddtetas }} \ddot{\theta}_{s}^{2}+\rho_{F} F_{a}^{2}\right) d t\right]
$$

As is recommended for multi objective optimization [13], different weighting values $\rho_{i}$ are included. In the case where the weighting values are modified, the optimization model becomes another case and will probably generate different optimal values. In general cases, these values are always considered constant for each simulation (optimization run), which is a function of the specific objective, if one prefers roll angle versus suspension deflection (or opposite). In this work a value of 10000 is used for the roll angle since its reduction is the main goal of the active roll control system. A weighting of 10 is given to the suspension deflection, 1 for angular acceleration and 0.000001 for the actuator force. This reduces the importance of power consumption and focuses on the maximum active system performances. These values express the results of many simulations that have been compiled and are also based on our own experience team. These choices have proven to be a good trade-off between comfort and road holding capability [10].

To use the ARE, performance index $\mathrm{J}$ is reformulated in matrix $\mathrm{Q}, \mathrm{R}$ and $\mathrm{N}$ with respect to Eq. (14).

$$
J=\frac{1}{T} E\left[\int x^{T} Q x+x^{T} N^{T} u+u^{T} N x+u^{T} R u d t\right]
$$




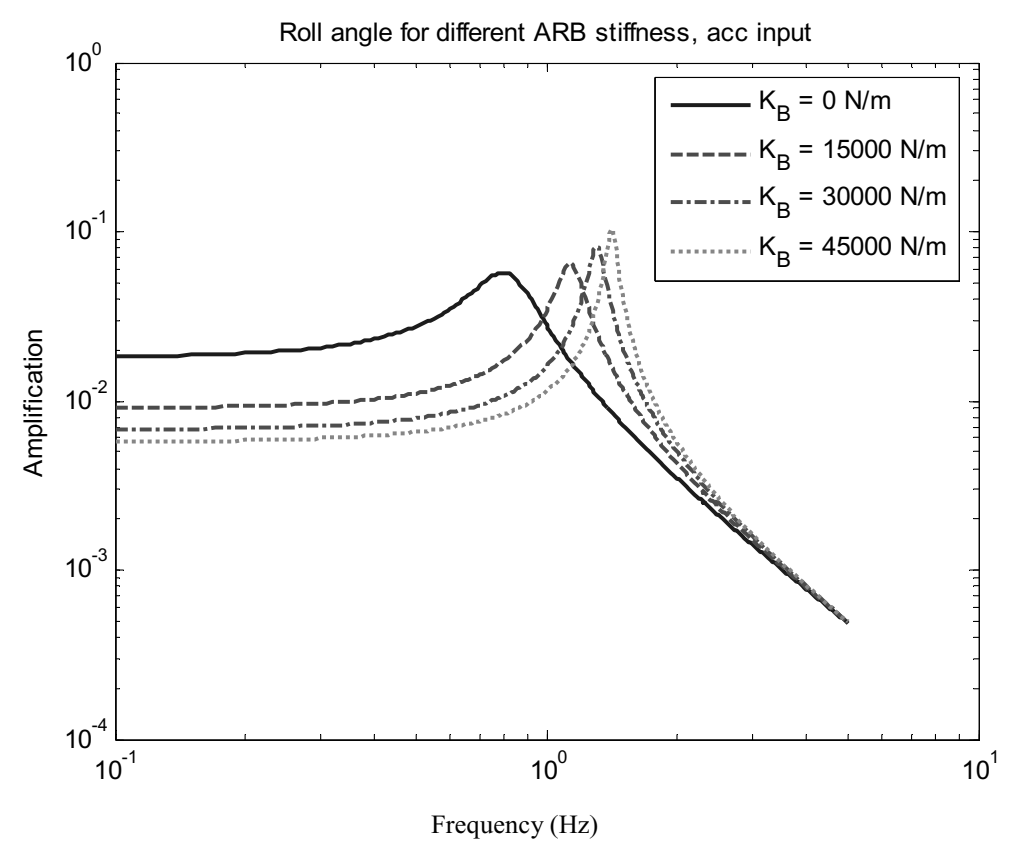

Fig. 5. Roll angle of passive suspension with passive ARB.

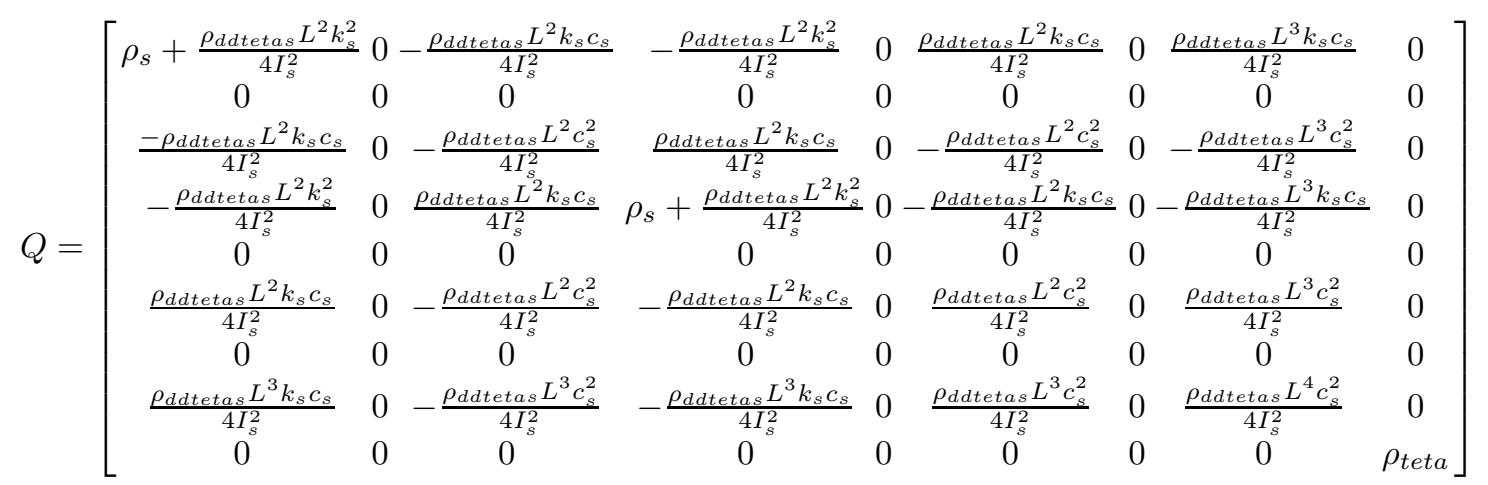

$N=\left[\begin{array}{c}-\frac{\rho_{\text {ddtetas }} L^{2} k_{s}}{2 I_{s}^{2}} \\ 0 \\ \frac{\rho_{\text {ddtetas }} L^{2} c_{s}}{2 I_{s}^{2}} \\ \frac{\rho_{\text {ddtetas }}^{2} k_{s}}{2 I_{s}^{2}} \\ 0 \\ -\frac{\rho_{\text {ddtetas }} L^{2} c_{s}}{2 I_{s}^{2}} \\ 0 \\ -\frac{\rho_{\text {ddtetas }} L^{3} c_{s}}{2 I_{s}^{2}} \\ 0\end{array}\right]$

$$
R=\frac{\rho_{\text {ddtetas }} L}{I_{s}}+\rho_{F}
$$

Equation (18) details the ARE and the modifications as indicated in [10,15]. Solution of the ARE is essential to calculate the control gain vector $\mathrm{G}$ that minimizes the performance index J. For this work the care function of Matlab 
has been used to solve the equation.

$$
\begin{aligned}
\bar{A} & =A-B R^{-1} N \\
\bar{Q} & =Q-N^{T} R^{-1} N \\
-\dot{\hat{M}} & =\hat{M} \bar{A}+\bar{A}^{T} \hat{M}-\hat{M} B R^{-1} B^{T} \hat{M}+\bar{Q} \\
\bar{G} & =R^{-1} B^{T} M \\
G & =R^{-1} B^{T} M+R^{-1} N
\end{aligned}
$$

Control vector $\mathrm{G}$ represents the optimal control law. For a given vector $\mathrm{G}$, the actuator force is calculated by multiplying $\mathrm{G}$ with the state vector $\mathrm{x}$.

$$
u=F_{a}=-G x=-\left[G_{1} x_{1}+G_{2} x_{2}+G_{3} x_{3}+G_{4} x_{4}+G_{5} x_{5}+G_{6} x_{6}+G_{7} x_{7}+G_{8} x_{8}\right]
$$

With the vehicle modeled here, we obtain the following control vector:

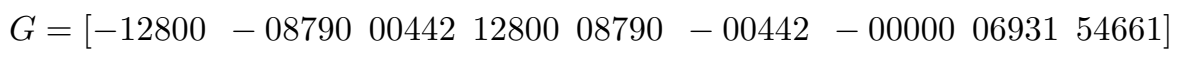

Once vector $\mathrm{G}$ is defined, a new matrix $\mathrm{A}_{c}$ can be constructed to model the system including the actuator and the controller [15]. This procedure is developed in Eq. (21).

$$
\begin{aligned}
& u=-G x \\
& \dot{x}=A x+B u+L_{r R} z_{r R}+L_{a} a_{\text {lat }} \\
& \dot{x}=A x-B G x+L_{r R} z_{r R}+L_{a} a_{l a t} \\
& \dot{x}=A_{C} x+L_{r R} z_{r R}+L_{a} a_{l a t} \\
& A_{C}=A-B G
\end{aligned}
$$

\section{Controller improvement}

The controller designed for the active system uses full state feedback control strategy. For this control method all the state variables have to be measured by electronic sensors. For the four DOF model, eight state variables are used requiring eight different sensors. The controller is labelled FSF8 and is used as a reference in the following graphs. Depending on the system, it can be hard or impossible to measure some of the variables. For example, tire deflection poses practical problems for instrumentation. In the literature [15] this problem is solved by using an optimal observer when state variables are not or cannot be measured. The observer allows the controller to estimate the value of unmeasured variables based on the system's parameters and dynamic characteristics.

The total system cost and complexity increases with the number of sensors, while reliability decreases. When using an optimal observer, the accuracy of the estimated variables is not guaranteed when the system parameters change. For an off-road vehicle this is a serious possibility since sprung mass depends on vehicle payload and suspension characteristics change with wear.

Since a reduction in the number of essential sensors is desirable while avoiding the use of an optimal observer, an attempt is made to simplify the controller. Realistic assumptions are made and tested with different gain combinations specified by the matrix G. This procedure will show the relative importance of the different gain in the control matrix.

The first step is an attempt to eliminate the tire deflection measurement. Because tire stiffness is higher than suspension stiffness, tire deflection should be small compared to suspension. Tire deflection gain is also smaller than suspension gain, so the part of the force related to tire deflection is expected to be small in comparison to suspension deflection and the other measured variables. For this reason the elimination of the tire deflection measurement is justified to reduce sensor numbers and to keep an acceptable performance. The number of sensors is then reduced from eight to six, labelled FSF6 in the following graphs.

Absolute unsprung mass velocity can be computed by integrating the signal of an accelerometer located on the unsprung mass. This is a viable solution in theory, but the integration of a noisy signal can lead to important errors as time increases. Tire deflection control gain is small compared to suspension deflection and roll angle. For this 
Table 3

Gain matrix

\begin{tabular}{lcrrrrrrrr}
\hline Matrix G & \multicolumn{1}{c}{$\mathrm{G}_{1}$} & \multicolumn{1}{c}{$\mathrm{G}_{2}$} & \multicolumn{1}{c}{$\mathrm{G}_{3}$} & \multicolumn{1}{c}{$\mathrm{G}_{4}$} & \multicolumn{1}{c}{$\mathrm{G}_{5}$} & \multicolumn{1}{c}{$\mathrm{G}_{6}$} & \multicolumn{1}{c}{$\mathrm{G}_{7}$} & $\mathrm{G}_{8}$ & $\mathrm{G}_{9}$ \\
\hline variable & $\mathrm{z}_{s} R^{-} \mathrm{z}_{u R}$ & $\mathrm{z}_{u R}-\mathrm{z}_{r} R$ & $\left(\mathrm{z}_{u R}\right)^{\prime}$ & $\mathrm{z}_{s L}-\mathrm{z}_{u L}$ & $\mathrm{z}_{u L}-\mathrm{z}_{r L}$ & $\left(\mathrm{z}_{u L}\right)^{\prime}$ & $\left(\mathrm{z}_{s}\right)^{\prime}$ & $\left(\operatorname{teta}_{s}\right)^{\prime}$ & teta $_{s}$ \\
FSF 8 & -12800 & -08790 & 00442 & 12800 & 08790 & -00442 & 0 & 06931 & 54661 \\
FSF 6 & -12800 & 0 & 00442 & 12800 & 0 & -00442 & 0 & 06931 & 54661 \\
FSF 4 & -12800 & 0 & 0 & 12800 & 0 & 0 & 0 & 06931 & 54661 \\
\hline
\end{tabular}

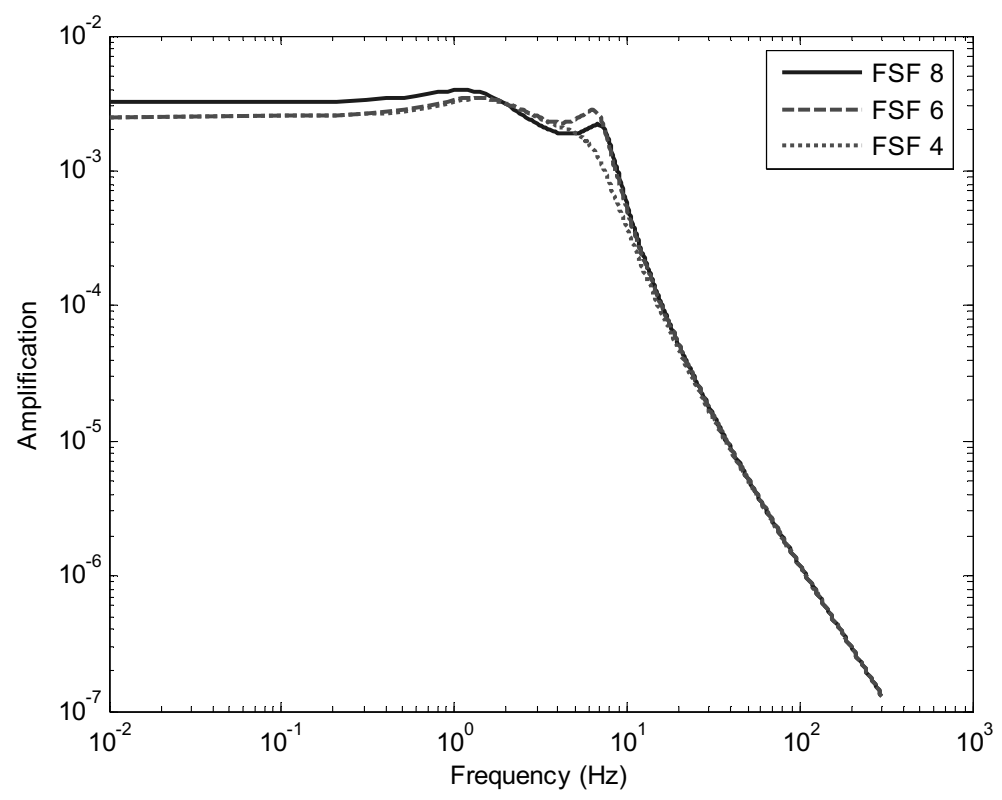

Fig. 6. Suspension deflection for lateral acceleration input.

reason a second hypothesis is proposed to reduce the number of sensors to four by eliminating the unsprung mass absolute speed measurement. This controller is labelled FSF4 in the following graphs. Right and left suspension deflection, roll angle and angular speed of the sprung mass are the reaming variables to be measured. These variables have an important control gain and are directly related to roll. For these reasons no attempt is made to eliminate any of them.

To evaluate the performance of each simplification, a control gain matrix corresponding to each possibility is defined in Table 3. Changing the control gain to 0 for the removed sensors does this. Figures 6 to 11 show the performances for the active system with eight (FSF 8), six (FSF 6) and four (FSF 4) measured variables.

For a lateral acceleration input the performance of the two simplified controllers is similar to the reference FSF8. As shown in Figs 6 and 7 the suspension deflection and roll angle is smaller in the low frequency range close to steady state for both simplified controllers. However, this is likely to result in a higher actuator force. At the unsprung mass resonance frequency suspension deflection is slightly reduced with the FSF4 but it is increased with the FSF6 controller. The roll angle is the same for all controllers at resonance and above. The angular acceleration slightly increases between 1 and $10 \mathrm{~Hz}$ using FSF6 and FSF4 but decreases for frequencies higher than $10 \mathrm{~Hz}$.

Because the lateral acceleration is caused by steering input, the frequency of the lateral acceleration input is limited to $5 \mathrm{~Hz}$. In this range the simplified controllers (FSF6 and FSF4) will be more stable than the optimal controller FSF8, but less comfortable in the 1 to $5 \mathrm{~Hz}$ range. Since low frequency roll angle and suspension deflection are reduced, a higher actuator force is expected in this range. Overall performance is nearly the same for all controllers for lateral acceleration input.

Figure 9 shows the suspension deflection for the left and right wheel in the case of right road motion input. For all controllers the right wheel response is similar but the left wheel response shows a difference. The simplified controllers have a slightly higher response between sprung and unsprung resonances but amplification is smaller for frequencies above unsprung resonance. 


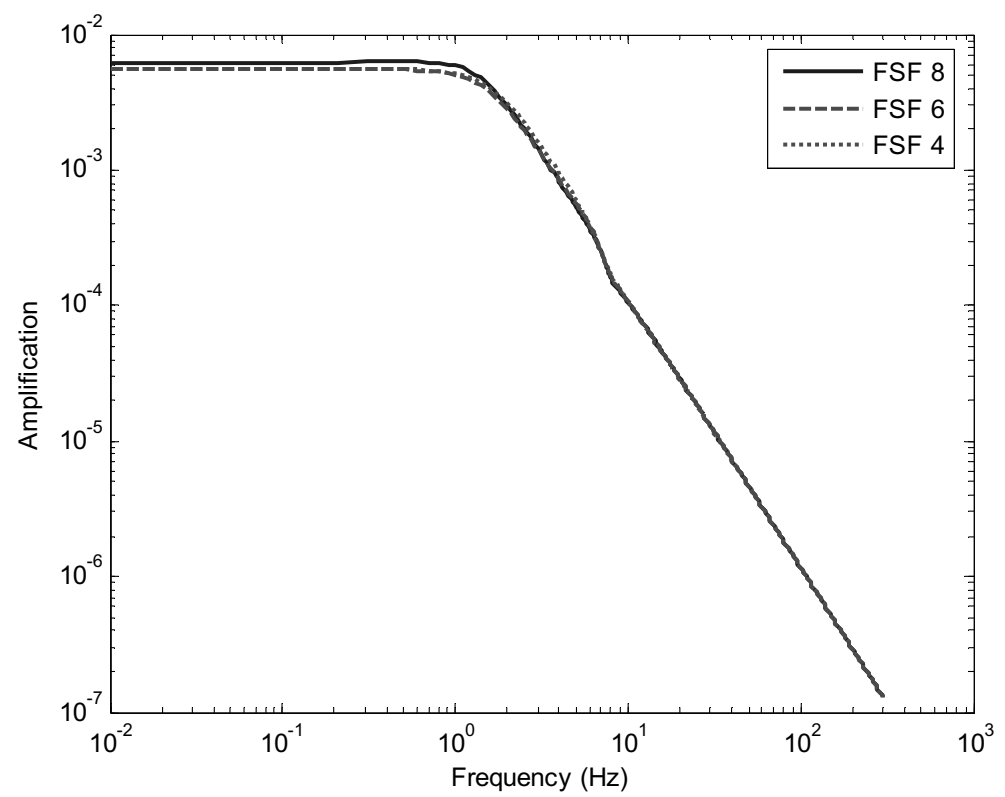

Fig. 7. Roll angle for lateral acceleration input.

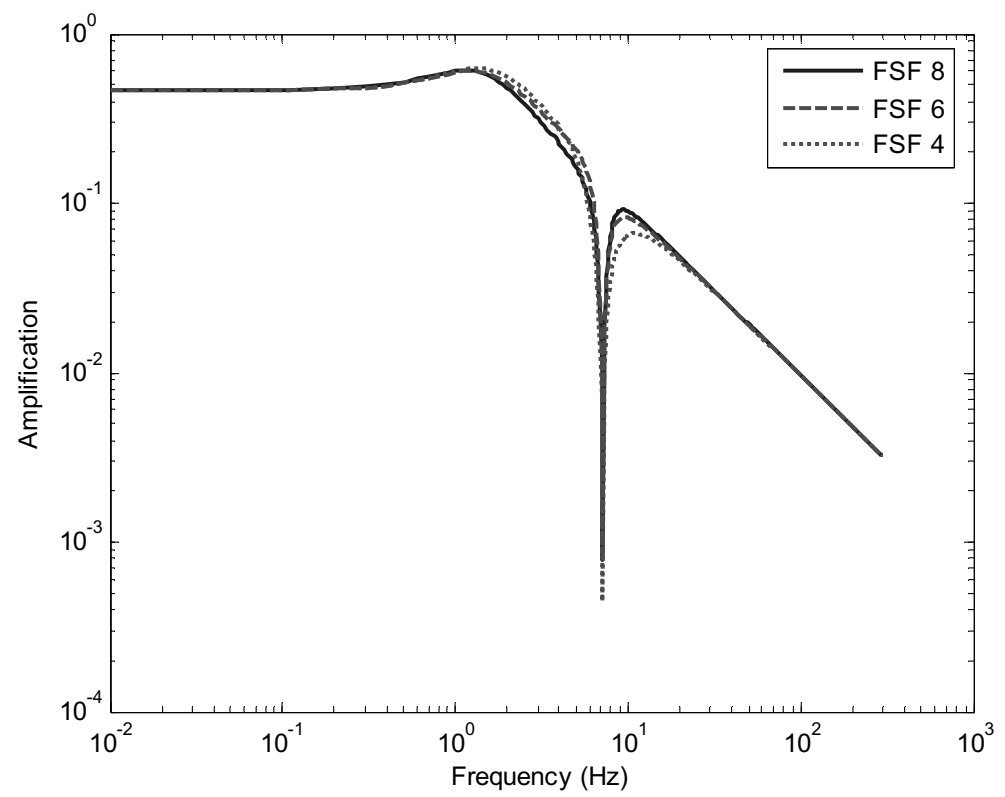

Fig. 8. Angular acceleration for lateral acceleration input.

Figure 10 shows a trade-off between low and high frequency for roll angle. For frequencies below the unsprung resonance the FSF8 is more efficient but looses efficiency when the frequency grows.

The main difference between the different controllers is the angular acceleration in case of road perturbation input as shown in Fig. 11. It is clear that FSF4 is less efficient between the sprung and unsprung resonance compared to FSF6 and FSF8. Since human beings are sensitive to acceleration in this frequency range, this is an important loss of performance for the active system.

When compared to the reference FSF8, the simplified controllers have a different gain for tire deflection. This 


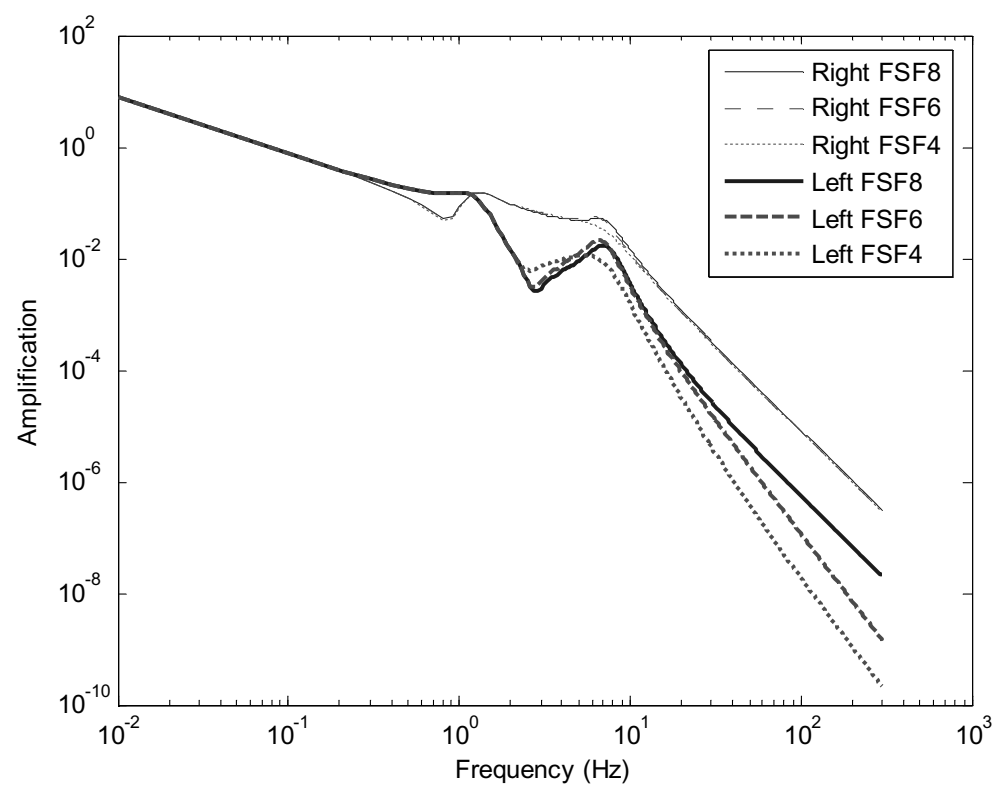

Fig. 9. Suspension deflection for right road input.

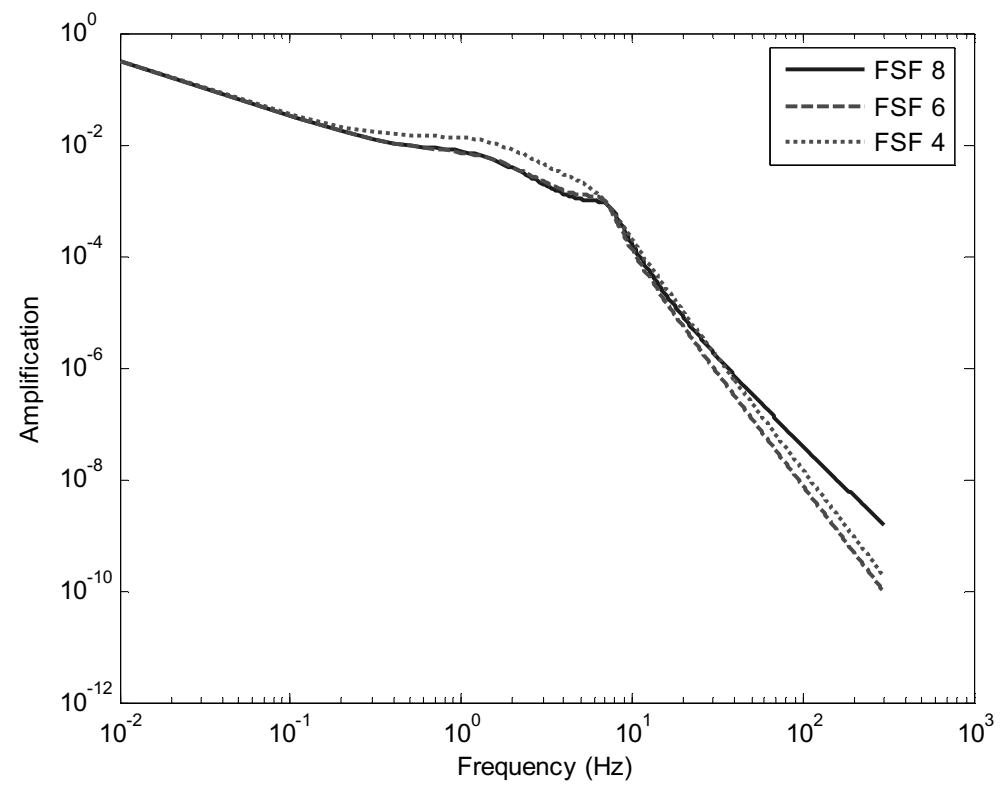

Fig. 10. Roll angle for right road input.

results in different tire stiffness for the FSF6 and FSF4 systems. The impact of tire stiffness is more important at unsprung resonance and above, as is visible on the response to road motion input. The overall difference between the FSF6 and the reference FSF8 is hardly noticeable for all criteria. This result confirms that tire deflection measurements have a negligible impact on performance. As a consequence, left and right tire deflection can be eliminated without significant reduction in the performance of the active system.

The FSF4 shows a similar response to the other active systems with the exception of angular acceleration. This difference can be explained by the elimination of the two terms associated with the unsprung mass velocity. These terms act like skyhook dampers; their elimination reduces damping in this zone, greatly reducing comfort around 


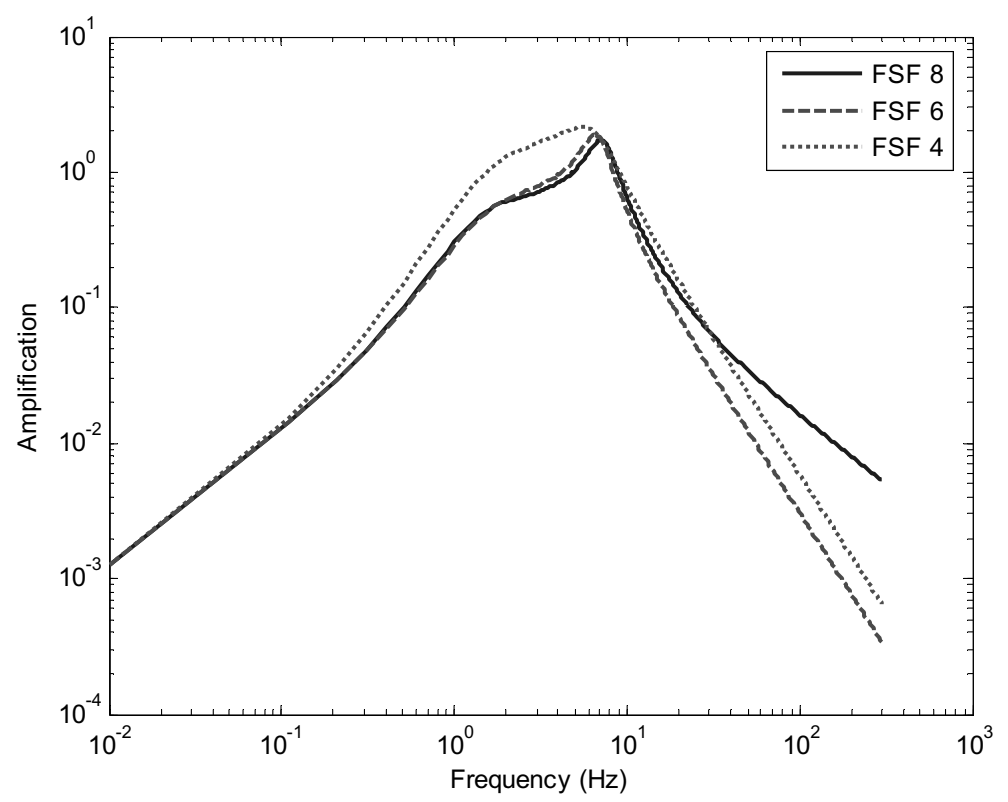

Fig. 11. Angular acceleration for right road input.

sprung mass resonance. From the results it is possible to use only four sensors FSF4 and reach a performance very close of the complete full state feedback control FSF8, with the exception of angular acceleration. For the FSF4, the four variables can be measured with three different sensors: one position sensor on each side of the vehicle to measure the distance between sprung and unsprung mass and one angle sensor on the sprung mass. The roll angle signal can be derived to measure the angular speed of the sprung mass. This reduction of the necessary number of sensors makes the active ARB system easier to implement on a real vehicle.

\section{Discussion and results}

To compare the performance of the different control systems the transfer function amplitude is plotted for each parameter (suspension deflection, sprung mass angle and angular acceleration) and for each input (lateral acceleration and right wheel perturbation). The passive suspension is defined by the values in Table 1. The passive ARB has a $7500 \mathrm{~N} / \mathrm{m}$ stiffness. The two simplified controllers are used to represent the active ARB since they have similar performance and reduced complexity compared to the reference controller. For the lateral acceleration input the graphs are plotted in Figs 12, 13 and 14.

As described above, the ARB greatly reduces the suspension deflection for low frequency since suspension stiffness is increased. Both passive systems show the same response for frequencies above resonance. The active ARB offers an important reduction of suspension deflection up to $2 \mathrm{~Hz}$. It is important to notice that the active system eliminates the resonance peak, decreasing the maximum amplitude of resonance. Since maximum suspension deflection is associated with high amplitude low frequency or medium amplitude lateral acceleration at resonance frequency, a noticeable reduction of the maximum suspension travel is expected. Suspension travel is critical for off-road vehicles making this a huge advantage for this kind of vehicle. Unfortunately, the active system is less efficient than the passive ones for frequencies above $2 \mathrm{~Hz}$.

Figure 13 shows the roll response to lateral acceleration input. The low frequency behaviour of all the different systems is similar to the suspension deflection since they are closely related. The resonance frequency in roll mode is higher for a suspension with an ARB than without an ARB because roll stiffness is higher. As for the suspension deflection, the active system is more efficient at frequencies below $2 \mathrm{~Hz}$ and eliminates the resonance peak. However, all the systems have the same response at frequencies higher than $2 \mathrm{~Hz}$, making the active system the best solution for this parameter. 


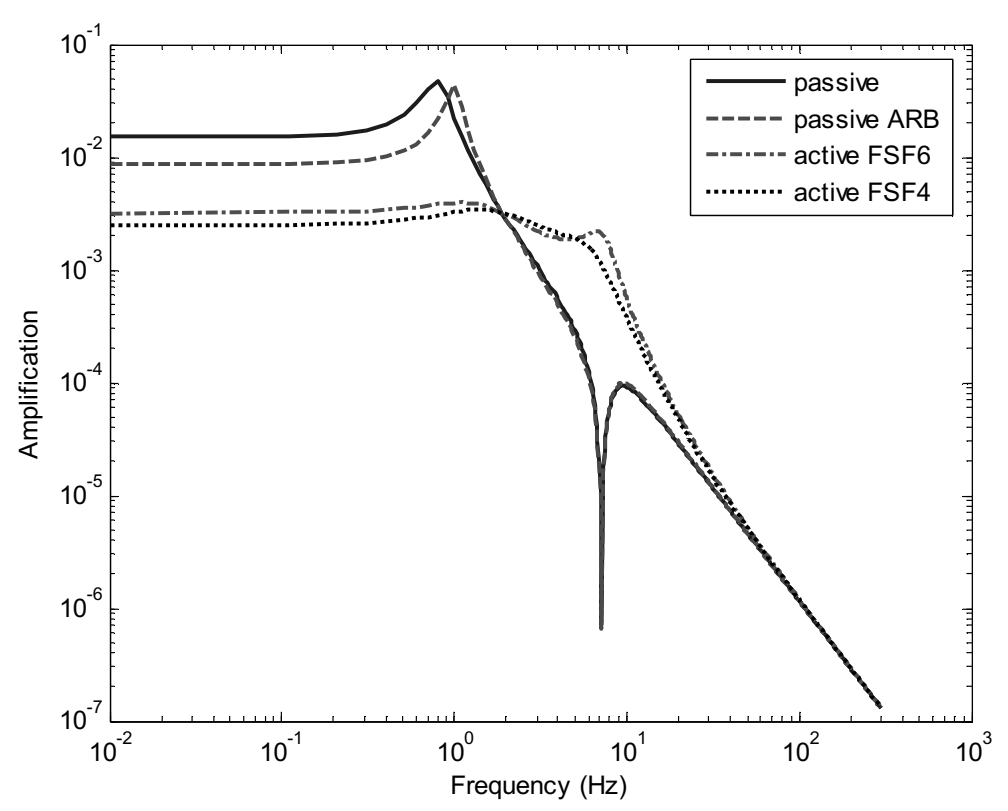

Fig. 12. Suspension deflection for lateral acceleration input.

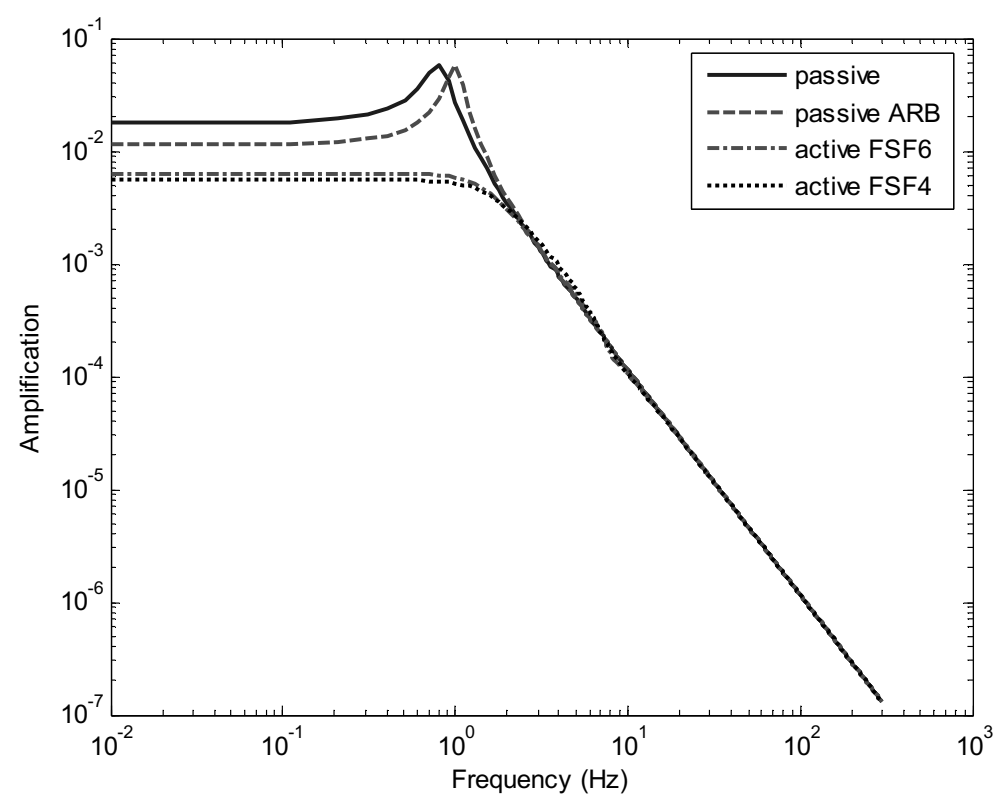

Fig. 13. Roll angle for lateral acceleration input.

Figure 14 shows the angular acceleration response for the different suspensions. For this criterion there is no difference between passive suspension, passive ARB and active ARB at low frequencies until resonance. The difference between passive suspension and passive ARB is only noticeable around resonance. The passive suspension has a higher damping coefficient and presents reduced amplitude compared to the passive ARB. As noticed on the two previous graphs the active system eliminates the resonance but has reduced performance above $2 \mathrm{~Hz}$.

For real operating conditions the lateral acceleration input is limited to low frequencies. The amplitude of the input starts to decrease at $1 \mathrm{~Hz}$ up to $5 \mathrm{~Hz}$ where it is hardly noticeable [15]. As a consequence, the higher frequencies 


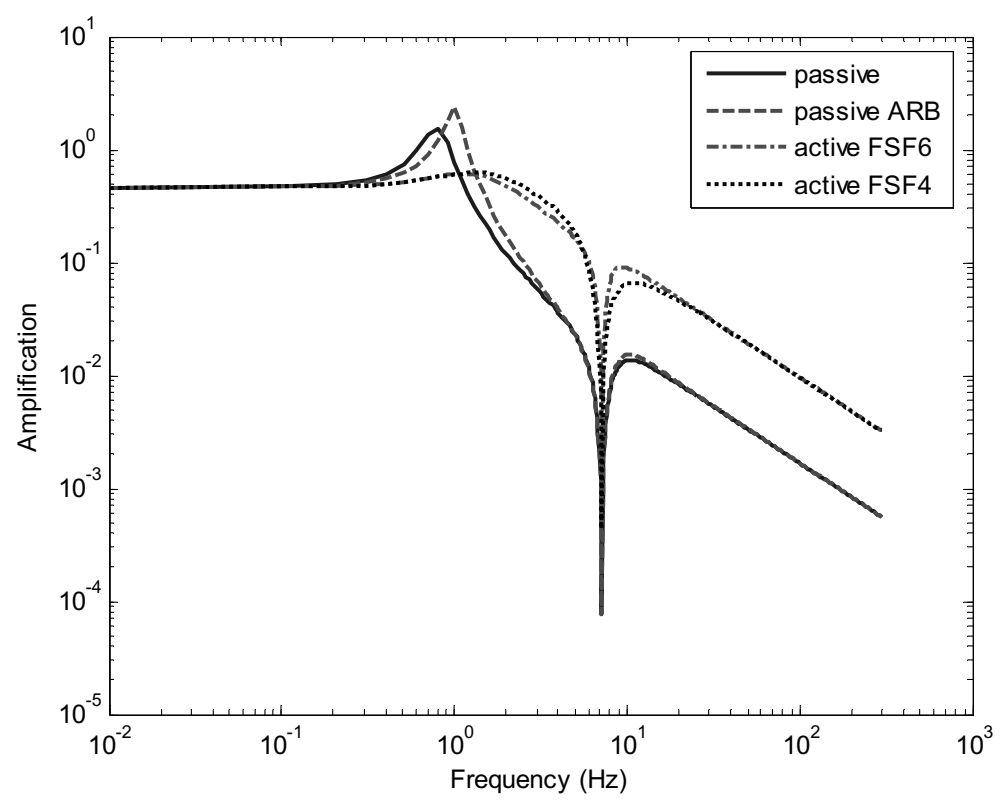

Fig. 14. Angular acceleration for lateral acceleration input.

have to be ignored because they will never be reached. In this case it is clear that the active ARB reduces the very low frequency response associated with steady state cornering. Most interestingly, the resonance noted in the passive systems is eliminated in the active ones. Considering all these advantages, the active system is more efficient than the passive ARB to reduce roll during cornering, thus improving stability and handling without decreasing comfort.

Figures 15, 16 and 17 show the suspension response for a road input perturbation.

Figure 15 shows the suspension deflection for the different systems in the case of road motion input at the right wheel while the left wheel isn't disturbed. The thin lines correspond to right suspension deflection and the thick ones to the left. Both passive systems have a smaller response on the left side compared to the right. As expected, the passive ARB shows a smaller deflection than the passive suspension. All four systems have a similar behaviour for the high frequencies but the active systems have a clearly higher amplification below $0.5 \mathrm{~Hz}$. If such frequencies were excited, the suspension travel would be too high for a real vehicle. This low frequency behaviour is explained by the travel required to reduce roll angle.

The roll angle response provides the explanation for the high suspension deflection observed in the previous graph. As we can see, the roll angle is highly reduced for the active systems compared to the passive ones. The road input case corresponds to a vertical displacement of the road/tire interface on the right side without any motion on the left side. For this input the active system reduces the sprung mass angle by increasing the distance between sprung and unsprung mass on one side and reducing it on the other. For a small amplitude input the required suspension displacement is acceptable but for low frequency inputs with associated high amplitude this requires larger suspension travel, as seen in Fig. 15.

As stated before, the right wheel is excited by the vertical motion of the road/wheel interface but the left wheel is assumed undisturbed, i.e. rolling on a perfectly flat surface. For a normal road this hypothesis is questionable since left and right wheel input aren't totally independent. For an off-road vehicle various obstacles could cause a low frequency high amplitude difference between right and left wheels. As shown in Figure 15 this would require an unrealistically large suspension travel, probably leading to suspension bottoming. Since the low frequency response of the active system is a potential problem for real condition use, two potential solutions will be proposed to reduce the chance of suspension bottoming.

Angular acceleration for road input is an important indication of passenger comfort on rough roads. As observable in Fig. 17, the passive ARB has an increased resonance peak and higher amplification between 1 and $10 \mathrm{~Hz}$ than the passive suspension. This is important since road input amplitude is noticeable and humans are sensitive to acceleration in this range. This confirms the reduction of comfort associated with the use of passive ARB. 


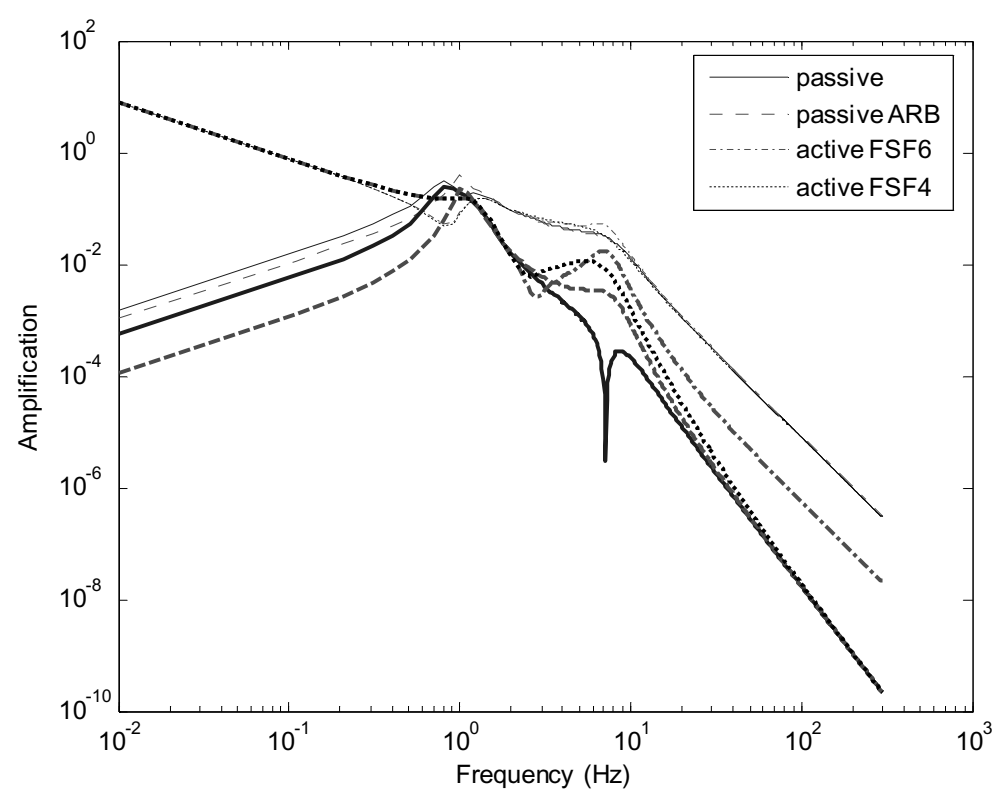

Fig. 15. Suspension deflection for right road input.

The two active systems show a high reduction in acceleration response up to $10 \mathrm{~Hz}$. As noted earlier, FSF6 provides more comfort in this sensitive zone than FSF4. This is the only noticeable difference between the two active systems. However FSF4 provides a better comfort than the two passive systems, making both active systems advantageous for passenger comfort.

The low frequency improvement is a consequence of the reduction in roll, which is unrealistic for frequencies below $0.5 \mathrm{~Hz}$. Fortunately this is outside the human sensitivity range and a major improvement will result in a reduction of acceleration between 0.5 and $10 \mathrm{~Hz}$.

These results demonstrate a similar response for the two passive systems studied. The two active systems show a similar performance and a great potential for improvement in different frequency ranges. For practical reasons and to maximise the benefits of the active system, the addition of filters is proposed.

The first consideration in the frequency response of the active system is the frequency response of the actuator. Obviously an active system will be inefficient if the actuator cannot operate properly. Most of the actuators available for the implementation of an active ARB are hydraulic or pneumatic. With such systems a delay is unavoidable in the application of the required force. This delay limits the maximum operating frequency of the actuator [16].

For a lateral acceleration input the benefits of the active systems are limited to frequencies below $2 \mathrm{~Hz}$. For a road motion input the active system is useful up to $10 \mathrm{~Hz}$ but most of the improvement occurs in the low frequency range below $2 \mathrm{~Hz}$. Since an average actuator has an operation frequency limit around $2 \mathrm{~Hz}$, a low pass filter with $2 \mathrm{~Hz}$ cutting frequency is recommended. The filter is inserted between the controller and the actuator. This ensures that the actuator is used in its working range and that the disadvantages of the active system at high frequencies are avoided. For a generic vehicle the cutting frequency will be a compromise between maximum actuator operating frequency and active system performance.

The second feature of the active system is the high suspension travel required for low frequency high amplitude road inputs. Figures 16 and 17 show the strong reduction of roll angle and angular acceleration using active roll control. This improvement implies that the actuator adjusts the sprung mass roll angle to keep the vehicle levelled, resulting in higher suspension deflection as discussed earlier. The first proposed solution is to add a high pass filter to disable the active system below $0.5 \mathrm{~Hz}$. This measure will ensure that the suspension response is similar to the passive system at low frequencies close to steady state. Since a large amount of force is required in this frequency range, the addition of such a filter will greatly reduce power consumption of the active system.

The use of a high pass filter will disable the active system (giving insufficient suspension travel). A second possibility is to allow the active system to work until suspension deflection becomes critical. This solution is a 


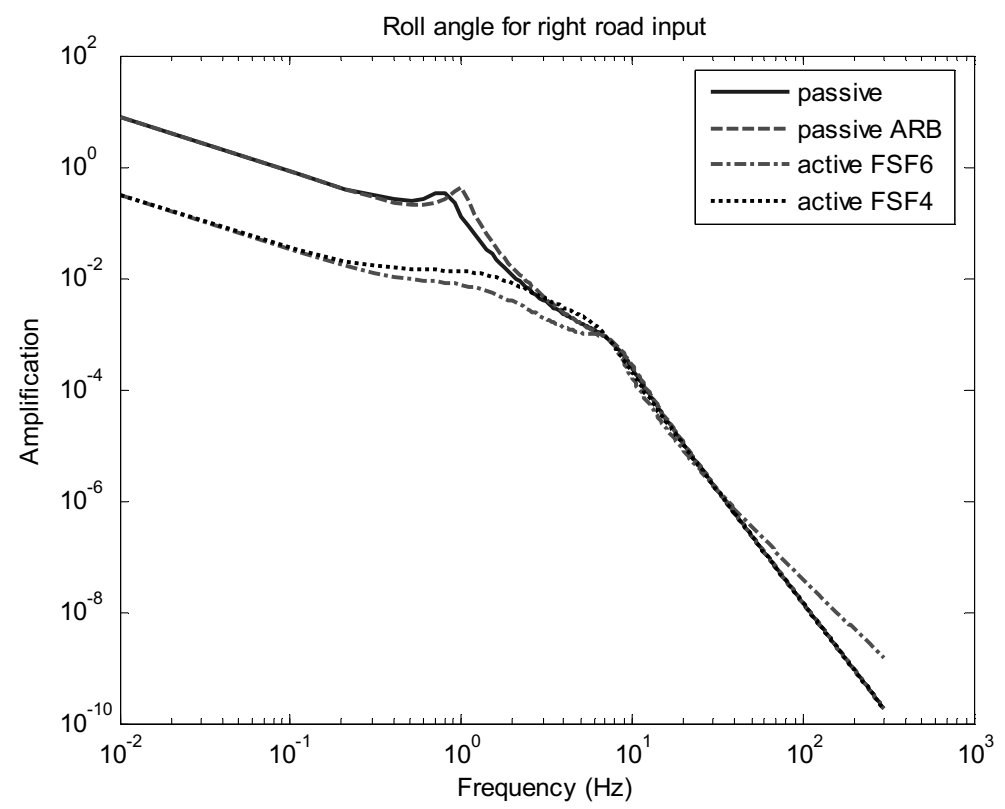

Fig. 16. Roll angle for right road input.

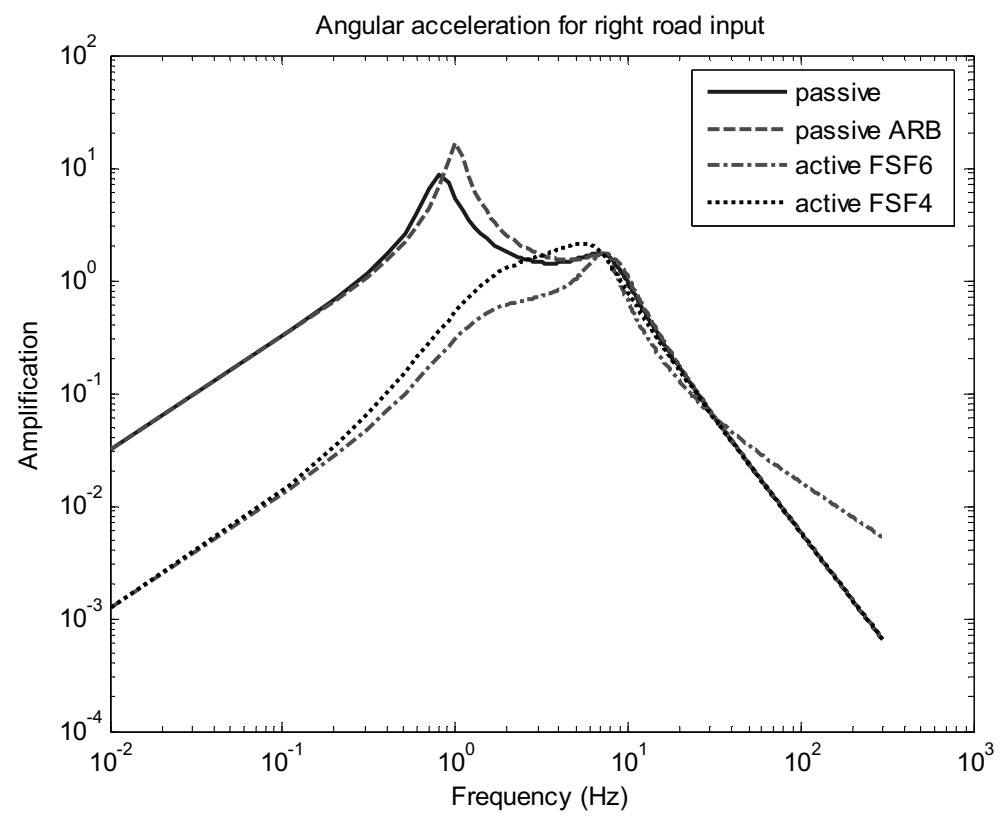

Fig. 17. Angular acceleration for right road input.

safety measure to avoid suspension bottoming while keeping the active system's advantages. Equation (22) gives an algorithm to adjust the total force depending on the suspension deflection.

$$
F_{a}=F_{a}\left(\frac{\left(z_{s i}-z_{u i}\right)_{\max }-\left|z_{s i}-z_{u i}\right|}{\left(z_{s i}-z_{u i}\right)_{\max }}\right)
$$

Naturally, depending on the suspension type and operating environment, other algorithms can be developed with smoother or sharper transitions. The value of the suspension deflection $\mathrm{z}_{s i}-\mathrm{Z}_{u i}$ is already measured by the sensors 
(for the force control) and the suspension travel $\left(\mathrm{z}_{s i}-\mathrm{Z}_{u i}\right)_{\max }$ is a fixed known value. Using this algorithm, the active system will use the required force to improve stability and comfort for relatively small suspension deflections. As suspension travel gets larger, the active force is reduced to the suspension deflection in a range close to the passive system.

\section{Conclusion}

This paper compares the performance of different types of suspensions: without ARB, with passive ARB and with an active ARB. A four DOF vehicle model has been used to model the suspension system of an off-road vehicle. The main goal of the study was to increase suspension stability by reducing the roll angle of the sprung mass without compromising passenger comfort.

A graphical method has been presented to select ARB stiffness based on selecting appropriate parameters. The active ARB controller has been designed by solving the ARE and a proposed simplification of the controller. The performances of the studied systems are analysed for suspension deflection, sprung mass roll angle and angular acceleration amplification.

It has been demonstrated that the use of a passive ARB increases stability but decreases comfort. This solution thus requires a compromise. However, the suggested active ARB design improves both the stability and comfort for the two inputs.

Results show that it is possible to ignore some state variables to reduce the number of essential sensors without major loss of performance. A controller using four different measurements proves to be more efficient than any passive system for the vehicle studied. Adding the measurement of the absolute velocity of the unsprung mass gives a better performance in case of road perturbation but poses practical instrumentation problems. The proposed reduction in the number of sensors eliminates the need for an optimal observer and reduces the total system cost.

The frequency response of the active system shows that major improvements are realised in the low frequency region. To maximize the active system's performance it is proposed to add a low pass filter between controller and actuator to limit the use of the active system to this range. Large improvements in roll angle will potentially require unrealistic suspension travel, mainly in the low frequency range below $0.5 \mathrm{~Hz}$. To avoid suspension bottoming, the use of a high pass filter or the addition of a control algorithm is suggested. It would maximize the benefits of the active ARB without causing suspension bottoming.

With these modifications the active system will provide a major improvement compared to the available passive suspension systems. The advantages are an increase in vehicle stability and passenger comfort for real world off-road use.

\section{Acknowledgments}

The work done in this study is sponsored by the Natural Science and Engineering Research Council of Canada (NSERC), CQRDA, FUQAC and CURAL. The authors wish to thank them for their support.

\section{References}

[1] D. Fischer and R. Isermann, Mechatronic semi active and active vehicle suspension, Control Engineering Practice 12 (2004), $1353-1367$.

[2] S. Hanba, T. Kawabe, O. Isobe, Y. Miyasato and Y. Watanabe, New semi-active suspension controller design using quasi-linearization and frequency shaping, Control Engineering Practice 6 (1998), 1183-1191

[3] D. Hrovat, Survey of Advanced Suspension Developments and Related Optimal Control Applications, Automatica 33(10) (1997), 17811817.

[4] T.J. Gordon, Non-Linear Optimal Control of a Semi-Active Vehicle Suspension, Chaos, Solitons, \& Fractals 5(9) (1995), $1603-1617$.

[5] H.-J. Kim and Y.-P. Park, Investigation of robust roll motion control considering varying speed and actuator dynamics, Mechatronics 14 (2004), 35-54.

[6] S.-M. Hwang and Y. Park, Active roll moment distribution based on predictive control, International Journal of Vehicle Design 16(1) (1995), 15 
[7] T. Hatano, M. Nagai, S. Takano and T. Tanigushi, Study on a vehicle dynamics model for improving roll stability, JSAE Review 24 (2003), $149-156$.

[8] J.C. Dixon, Tires, Suspension and Handling, Warrendale: Society of Automotive Engineers, Inc, $1996,621$.

[9] T.D. Gillespie, Fundamentals of Vehicle Dynamics, Warrendale: Society of Automotive Engineers, Inc, $1992,495$.

[10] M. Bouazara, An Analytical Method for Optimal Suspension Parameters and Tire Forces Analysis for a Vehicle Model. Proceedings of Canadian Society of Mechanical Engineering, Queen's University, Kingston, Ontario, May 21-24, 2002, ISBN 0-9730900-1-4.

[11] P. Holmlund, R. Lundström and N.J. Mansfield, Comparison of subjective responses to vibration and shock with standard analysis methods and absorbed power, Journal of Sound and Vibration 230(3) (2000), 477-491.

[12] R.M. Chalassani, Ride performance potential of active suspension system part I: Simplified analysis based on a quarter-car model. Symposium on simulation and control of ground vehicle and transportation systems: AMD-80(DSC.2), 1986, 187-204

[13] M. Blundell and D. Harty, The Multibody Systems Approach to Vehicle Dynamics, Warrendale: Society of Automotive Engineers, 2004, 518.

[14] T.J. Tsao and R. Chen, The design of an active suspension force controller using genetic algorithms with maximum stroke constraints, Journal of automobile engineering 15(D3) (2001), 317-327.

[15] B. Friedland, Control System Design, United States: McGraw-Hill, 1976, 513.

[16] I. Cech, Anti-roll and active roll suspensions, Vehicle System Dynamics 33(2) (2000), 91. 

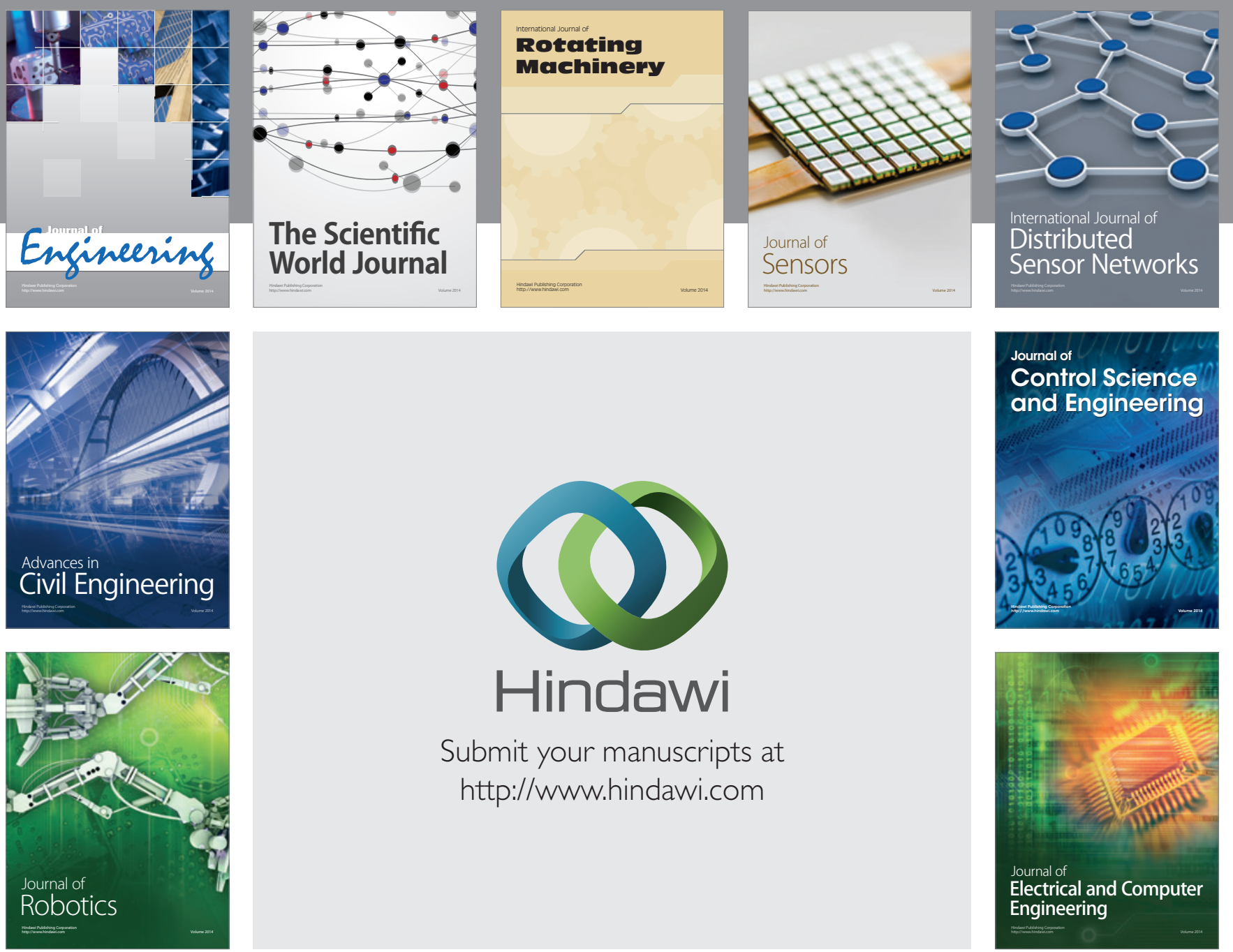

Submit your manuscripts at

http://www.hindawi.com
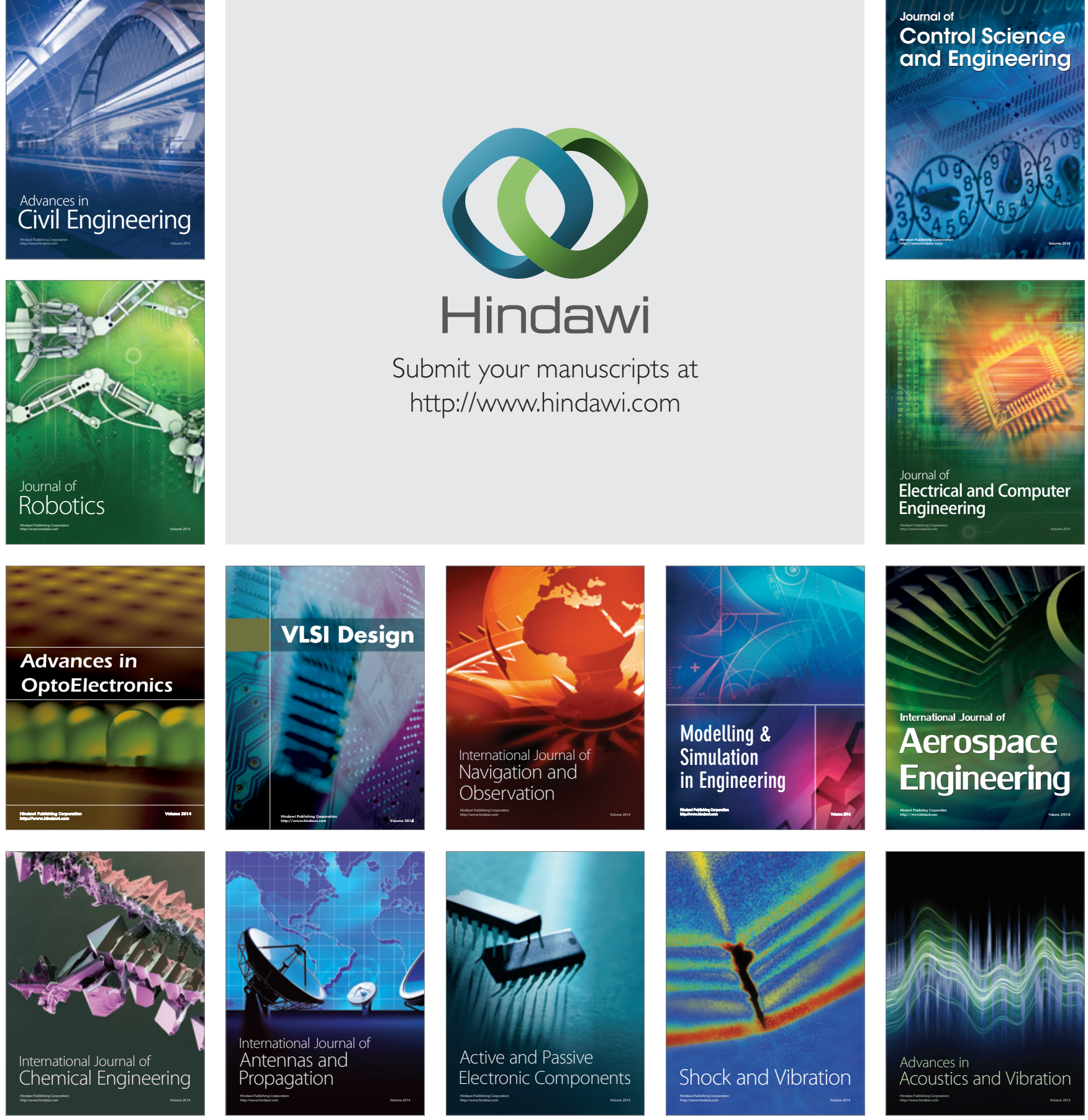